\title{
A Retrospective Comparative Study between Levosimendan and Adrenaline as a Pharmacological Protocol for the Management of Coronary Artery Bypass Grafting Patients with Low Ejection Fraction: A Friend or Foe
}

\author{
Mohammed Nabil Abd Al Jawad1*, Mohammed S. Shorbagy² \\ ${ }^{1}$ Department of Cardiothoracic Surgery, Ain Shams University, Cairo, Egypt \\ ${ }^{2}$ Department of Anesthesiology, Ain Shams University, Cairo, Egypt \\ Email: *mohammed_abdaljawad@med.asu.edu.eg
}

How to cite this paper: Al Jawad, M.N.A. and Shorbagy, M.S. (2018) A Retrospective Comparative Study between Levosimendan and Adrenaline as a Pharmacological Protocol for the Management of Coronary Artery Bypass Grafting Patients with Low Ejection Fraction: A Friend or Foe. World Journal of Cardiovascular Surgery, 8, 219231.

https://doi.org/10.4236/wjcs.2018.811022

Received: October 29, 2018

Accepted: November 23, 2018

Published: November 26, 2018

Copyright ( $\odot 2018$ by authors and Scientific Research Publishing Inc. This work is licensed under the Creative Commons Attribution International License (CC BY 4.0).

http://creativecommons.org/licenses/by/4.0/

\section{c) (†) Open Access}

\begin{abstract}
Background: Left ventricular ejection fraction is an independent determinant of the outcome of coronary artery bypass surgery. Low preoperative ejection fraction requires special care in terms of pharmacological and mechanical inotropic support. Adrenaline is the most widely used inotropic drug, while levosimendan is a relatively new inotropic drug in the field of cardiac surgery. In this study, we aimed to evaluate the relative efficacy of levosimendan in low ejection fraction patients undergoing coronary artery bypass grafting (CABG). Methods: A retrospective comparative study was performed with 63 patients who underwent isolated on-pump elective CABG with a preoperative ejection fraction below $40 \%$. Patients were allocated to the adrenaline group $(\mathrm{n}=35)$ and levosimendan group $(\mathrm{n}=28)$. Patients were further stratified according to ejection fraction above $30 \%$ and below or equal to $30 \%$. The primary outcome was cardiac-related mortality, while other parameters were considered secondary endpoints. Results: EuroSCORE of the adrenaline group was $3.34 \pm 1.26$ and for the levosimendan group $3.15 \pm 1.42$ ( $\mathrm{p}$ value 0.576). Nine patients of the adrenaline group had new postoperative atrial fibrillation compared to seven patients in the levosimendan group ( $\mathrm{p}$ value 0.948). Two patients of the adrenaline group had postoperative ventricular arrhythmia compared to only one patient in the other group ( $\mathrm{p}$ value 0.691 ). The adrenaline group had higher doses of inotropic support compared to the levosimendan group $210.84 \pm 23.74$ and $157.4 \pm 22.69 \mathrm{ng} / \mathrm{kg} / \mathrm{min}$ respectively ( $\mathrm{p}$ value $<0.001$ ). Longer ventilation hours and overall duration of ICU stay
\end{abstract}


were also noticed in the adrenaline group $32.57 \pm 7.23$ hours, $8.84 \pm 3.28$ days in comparison to the levosimendan group $24.37 \pm 5.09$ hours, $6.23 \pm 2.37$ days ( $\mathrm{p}$ values $<0.001$ and 0.002 respectively). However, the primary endpoint was not significantly different between the two groups. Conclusions: The levosimendan-based protocol failed to improve overall mortality in low ejection fraction patients undergoing CABG. However, this protocol significantly reduced the dose of inotropic and vasoconstrictor support needed, ventilation hours and duration of ICU stay.

\section{Keywords}

Levosimendan, Epinephrine, Low Ejection Fraction, Coronary, Adrenaline

\section{Introduction}

Coronary artery bypass grafting (CABG) is the most common cardiac surgery in adults at present [1]. Various factors, mainly the perioperative left ventricular ejection fraction, degree of ischemia and coronary lesion anatomy, contribute to the outcome of this procedure [2]. Among other factors are the insertion of an intra-aortic balloon pump (IABP) and a low cardiac output status perioperatively [3].

The use of intra-aortic counterpulsion is debatable with regard to the timing of insertion. Although many studies have found that preoperative insertion has a beneficial effect because it stabilizes the hemodynamics, increases coronary perfusion and reduces myocardial ischemia [4]. However, the recent guidelines of the European Society of Cardiology (ESC) do not recommend the routine use of IABP in a preoperative setting [class III] [5].

Following cardiac surgery, myocardial contractility tends to decrease owing to myocardial edema and decreased myocardial compliance. This process continues to occur in the early postoperative period, which requires careful and delicate pharmacologic management in patients already suffering from depressed left ventricular function [4].

At our institute, we use a combination of inotropic adrenaline infusion with coronary dilator glyceryl trinitrate (GTN) infusion in most patients as a standard protocol in addition to mechanical support in the early postoperative period. More recently, some surgeons and anesthetists introduced the new inotropic drug levosimendan combined with titrated doses of noradrenaline infusion as an alternative to the well-established protocol.

In this study, we aimed to compare the two protocols in terms of mortality and the associated low cardiac output syndromes in the early postoperative period.

\section{Patients and Methods}

A retrospective study of the registry of the Department of Cardiothoracic Sur- 
gery at our institution was conducted from January 2015 until December 2017. The study included 63 patients of both sexes who had undergone elective, isolated, on-pump CABG for three-vessel disease (regardless of the final number of grafts) utilizing antegrade blood-enriched cardioplegic arrest under moderate hypothermia $28^{\circ} \mathrm{C}-32^{\circ} \mathrm{C}$. The preoperative left ventricular ejection fraction was below $40 \%$ by two-dimensional echocardiography, and the left ventricular diastolic diameter was up to $6.5 \mathrm{~cm}$. We excluded patients with mitral regurgitation grade III or IV, patients who underwent mitral valve intervention, and patients with other comorbidities of hepatic, renal or respiratory origin.

We stratified the patients into two groups according to ejection fraction below or equal to $30 \%$ or above $30 \%$.

- The adrenaline/GTN protocol: 35 patients received this initial protocol and were titrated as needed.

a) Adrenaline IV infusion at a starting dose of $50-100 \mathrm{ng} / \mathrm{kg} / \mathrm{min}$ (Adrenaline; $1 \mathrm{mg} / 1 \mathrm{ml}$ ampoule, Chemical Industries Development "CID”, Giza, Egypt)

b) Glyceryl trinitrate IV infusion at a starting dose of $0.5-1 \mu \mathrm{g} / \mathrm{kg} / \mathrm{min}$ IV infusion (Nitronal Aqueous; $1 \mathrm{mg} / \mathrm{ml}$ solution, Sunny Pharmaceutical, G. PohlBoskamp GmbH \& Co.KG, Germany).

- The levosimendan/noradrenaline protocol: 28 patients initially received this protocol.

a) Levosimendan IV infusion at a loading dose of $12 \mu \mathrm{g} / \mathrm{kg}$ intravenously for $10 \mathrm{~min}$, followed by intravenous infusion $(0.1-0.2 \mu \mathrm{g} / \mathrm{kg} / \mathrm{min})$ for 24 hours (Simdax; Orion Pharma, Finland Orion Corporation, Orionintie, Espoo, Finland) and low-dose GTN infusion the next day.

b) Noradrenaline IV infusion at a starting dose of $30-50 \mathrm{ng} / \mathrm{kg} / \mathrm{min}$ (levophrine; norepinephrine bitartrate $8 \mathrm{mg} / 4 \mathrm{ml}$ solution, Alexandria Co. for pharmaceuticals for Egypharma, Egypt).

The primary endpoint of the study was in-hospital mortality. Secondary endpoints included the presence of low cardiac output syndrome, reventilation due to a cardiac cause, prolonged use of inotropic and vasoconstrictor support, the need for adjuvant inotropic or vasoconstrictor support or an IABP, ventilation hours, ICU stay hours, and total hospital stay.

\section{Statistical Analysis}

The statistical presentation and analysis were conducted using the mean and standard deviation; unpaired Student's t-test was used to compare quantitative data between two groups, and chi-squared tests were computed for $2 \times 2$ tables using qualitative data by IBM SPSS Statistics for Windows, Version 20.0. Armonk, NY: IBM Corp.).

\section{Results}

The study included 63 patients, 35 of whom belonged to the adrenaline protocol. The majority of patients in both groups were male. The patients' demographics, 
associated comorbidities, and Euro score average are shown in Table 1.

The preoperative ejection fraction was comparable in both the adrenaline and levosimendan groups at $29.45 \pm 3.75$ and $30.67 \pm 4.28$, respectively. Five patients in each of the two groups had left main disease. Six patients in the adrenaline group underwent preoperative insertion of an IABP, while only five had it inserted in the other group. Preoperative data are shown in Table 1.

Operative details along with the final number of grafts are shown in Table 1.

The overall postoperative course is shown in Table 1, and the overall incidence of postoperative arrhythmia was higher in the adrenaline group than in the levosimendan group, but the difference was not statistically significant. The overall use of postoperative IABP was higher in the adrenaline group than in the levosimendan group, but the difference was not statistically significant. The need for inotropic support was higher in the levosimendan group than in the adrenaline group, but the difference was not statistically significant. The dose of adjuvant adrenaline use in the levosimendan group was significantly lower than the dose used in the adrenaline group (p-value $<0.001$, highly significant). Similarly, the dose of noradrenaline in the levosimendan group was significantly lower than that in the adrenaline group. The total ventilation hours and, subsequently, the duration of ICU stay were significantly lower in the levosimendan group than in the adrenaline group. The primary outcome regarding mortality in the two groups was not different (Table 1).

A comparative hemodynamic study regarding the heart rate showed lower heart rate values in the levosimendan group than in the adrenaline group, but the difference was not statistically significant on Day 0 or the following day (Table 2, Figure 1).

The values of systolic blood pressure failed to show a significant difference in either group even after 48 hours (Table 3, Figure 2).

The diastolic blood pressure values were not significantly different in either group (Table 4, Figure 3 ).

When further stratifying the patients according to their preoperative left ventricular (LV) ejection fraction, both groups showed a significant difference in the doses of adrenaline and noradrenaline, with significantly higher doses in the adrenaline group than in the statistical significance being higher in the adrenaline group than in the levosimendan group. Again, the mortality in both groups failed to exhibit a statistically significant difference (Table 5, Table 6, Figure 4).

\section{Discussion}

Preoperative left ventricular function (EF) is one of the independent risk factors determining the outcome of coronary artery surgery. Operating in this category of patients, i.e., patients with an $\mathrm{EF}<40 \%$, carries a higher incidence of mortality and morbidity than operating on patients with normal ejection fraction [6].

Preoperative EF is included in most "scoring systems,", e.g., EuroSCORE and STS risk calculator, as it is the strongest predictor of postoperative mortality, low 
Table 1. Demographics, associated comorbidities, preoperative data, operative details and postoperative course.

\begin{tabular}{|c|c|c|c|c|}
\hline & $\begin{array}{c}\text { Adrenaline group } \\
\mathrm{n}=35\end{array}$ & $\begin{array}{c}\text { Levosimendan } \\
\text { group } \mathrm{n}=28\end{array}$ & $\mathrm{X}^{2} / \mathrm{t}$ & $\mathrm{P}$-value \\
\hline Age & $51.45 \pm 4.37$ & $52.08 \pm 5.12$ & 0.527 & 0.600 \\
\hline \multicolumn{5}{|l|}{ Sex } \\
\hline Female & 5 & 7 & \multirow{2}{*}{1.158} & \multirow{2}{*}{0.282} \\
\hline Male & 30 & 21 & & \\
\hline BMI & $25.5 \pm 4.16$ & $26.12 \pm 5.31$ & 0.520 & 0.605 \\
\hline Weight $(\mathrm{kg})$ & $73.4 \pm 13.71$ & $72.78 \pm 11.7$ & 0.190 & 0.849 \\
\hline Height $(\mathrm{cm})$ & $169.1 \pm 8.55$ & $168.56 \pm 7.62$ & 0.261 & 0.794 \\
\hline $\mathrm{DM}$ & 19 & 12 & 0.813 & 0.367 \\
\hline Systemic hypertension & 19 & 15 & 0.003 & 0.955 \\
\hline NYHA I & 2 & 2 & 0.053 & 0.817 \\
\hline NYHA II & 10 & 8 & 0.000 & 1.000 \\
\hline NYHA III & 12 & 11 & 0.168 & 0.682 \\
\hline NYHA IV & 11 & 7 & 0.315 & 0.575 \\
\hline Angina & 5 & 4 & 0.000 & 1.000 \\
\hline Previous recent MI & 10 & 8 & 0.000 & 1.000 \\
\hline EuroSCORE (range $1.11-6.34$ ) & $3.34 \pm 1.26$ & $3.15 \pm 1.42$ & 0.562 & 0.576 \\
\hline $\mathrm{EF}$ (range 25 - 39)\% & $29.45 \pm 3.75$ & $30.67 \pm 4.28$ & 1.628 & 0.109 \\
\hline ESD (range $3.5-5.5$ ) cm & $4.2 \pm 1.14$ & $3.95 \pm 1.45$ & 0.766 & 0.446 \\
\hline EDD (range $4.2-6.5) \mathrm{cm}$ & $5.13 \pm 1.22$ & $5.07 \pm 1.36$ & 0.184 & 0.854 \\
\hline Left main disease & 5 & 5 & 0.149 & 0.700 \\
\hline Preoperative IABP & 6 & 5 & 0.006 & 0.941 \\
\hline Preoperative inotropes & 0 & 0 & 0.000 & 1.000 \\
\hline Total bypass (range $70-120$ ) $\mathrm{min}$ & $94.23 \pm 5.67$ & $95.12 \pm 4.84$ & 0.660 & 0.512 \\
\hline ACC time (range $45-85$ ) $\mathrm{min}$ & $68.37 \pm 6.69$ & $70.08 \pm 5.66$ & 1.078 & 0.285 \\
\hline Arterial grafts & 1 & 1 & 0.026 & 0.872 \\
\hline Venous grafts (range $1-3$ ) & $1.8 \pm 0.67$ & $1.94 \pm 0.43$ & 0.958 & 0.342 \\
\hline Total grafts (range $2-4$ ) & $2.87 \pm 1.01$ & $3.12 \pm 0.45$ & 1.215 & 0.228 \\
\hline Postoperative AF & 9 & 7 & 0.004 & 0.948 \\
\hline Postoperative ventricular arrhythmia & 2 & 1 & 0.158 & 0.691 \\
\hline Need for postoperative IABP & 4 & 1 & 1.314 & 0.252 \\
\hline Need for inotropes $>48$ hours & 10 & 13 & 2.140 & 0.144 \\
\hline $\begin{array}{l}\text { Need for readministration of } \\
\text { inotropes }\end{array}$ & 7 & 3 & 1.004 & 0.316 \\
\hline Need for reintubation & 4 & 1 & 1.314 & 0.252 \\
\hline Use of adjuvant adrenaline & Not applicable & 20 & - & - \\
\hline
\end{tabular}




\section{Continued}

\begin{tabular}{ccccc}
\hline $\begin{array}{c}\text { Dose of adrenaline for all patients } \\
(\mathrm{ng} / \mathrm{kg} / \mathrm{min})\end{array}$ & $210.84 \pm 23.74$ & $157.4 \pm 22.69$ & 9.053 & $<0.001^{* *}$ \\
$\begin{array}{c}\text { Use of adjuvant noradrenaline } \\
\text { Dose of noradrenaline for all patients } \\
(\mathrm{ng} / \mathrm{kg} / \mathrm{min})\end{array}$ & 6 & Not applicable & - & - \\
$\begin{array}{c}\text { Total ventilation hours } \\
\text { ICU stay days }\end{array}$ & $32.57 \pm 7.23$ & $24.37 \pm 5.09$ & 5.075 & $<0.001^{* *}$ \\
Hospital stay days & $8.84 \pm 3.28$ & $6.23 \pm 2.37$ & 3.534 & $0.002^{*}$ \\
Death & $10.67 \pm 3.57$ & $9.26 \pm 2.33$ & 1.804 & 0.076 \\
\hline
\end{tabular}

$\mathrm{BMI}=$ Body mass index, $\mathrm{DM}=$ diabetes mellitus, $\mathrm{NYHA}=$ New York Heart Association, $\mathrm{MI}=$ myocardial infarction, $\mathrm{EF}=$ ejection fraction, $\mathrm{ESD}=$ end-systolic diameter, $\mathrm{EDD}=$ end-diastolic diameter, $\mathrm{IABP}=\mathrm{in}$ tra-aortic balloon pump, ACC $=$ aortic cross clamp, AF $=$ atrial fibrillation. Data are shown as the mean \pm $\mathrm{SD}$, * statistically significant, ** statistically highly significant.

Table 2. Hemodynamic comparative study of heart rate (beats/minute)

\begin{tabular}{ccccc}
\hline Hemodynamic study of heart rate & Adrenaline & Levosimendan & $\mathrm{t}$ & P-value \\
\hline Preoperation & $82.09 \pm 9.29$ & $81.57 \pm 8.56$ & 0.229 & 0.820 \\
Day 0 & $106.14 \pm 10.35$ & $101.37 \pm 7.59$ & 2.038 & $0.046^{*}$ \\
After 24 hours & $98.01 \pm 10.50$ & $92.42 \pm 8.37$ & 2.293 & $0.025^{\star}$ \\
After 48 hours & $91.51 \pm 10.06$ & $89.68 \pm 8.46$ & 0.769 & 0.445 \\
Prior to hospital discharge & $86.80 \pm 7.11$ & $84.07 \pm 7.51$ & 1.477 & 0.145 \\
\hline
\end{tabular}

Data are shown as the mean $\pm \mathrm{SD}$, ${ }^{*}$ statistically significant.

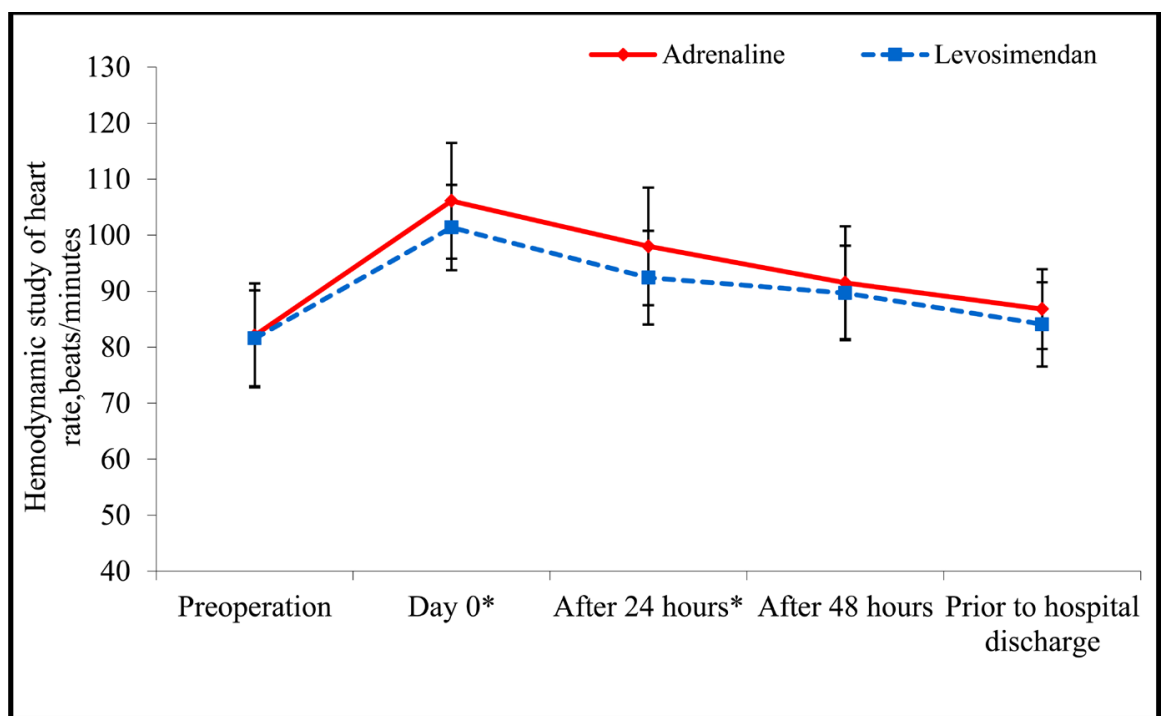

Figure 1. Hemodynamic comparative study of heart rate, ${ }^{\star}$ statistically significant.

cardiac output, the need for inotropic support, acute renal failure, prolonged ventilation and chest infection, and prolonged ICU and hospital stay [7].

Pharmacological inotropic support includes three subtypes: catecholamines, phosphodiesterase inhibitors, and calcium sensitizers. Most of these drugs act by 
Table 3. Hemodynamic comparative study of systolic blood pressure (mmHg).

\begin{tabular}{ccccc}
\hline $\begin{array}{c}\text { Hemodynamic study of systolic } \\
\text { blood pressure }\end{array}$ & Adrenaline & Levosimendan & $\mathrm{t}$ & P-value \\
\hline Preoperation & $125.33 \pm 11.19$ & $125.12 \pm 10.57$ & 0.076 & 0.939 \\
Day 0 & $122.91 \pm 16.85$ & $124.26 \pm 9.78$ & 0.376 & 0.708 \\
After 24 hours & $127.99 \pm 25.05$ & $130.29 \pm 23.68$ & 0.371 & 0.712 \\
After 48 hours & $125.45 \pm 8.06$ & $130.56 \pm 9.31$ & 2.334 & $0.023^{*}$ \\
Prior to hospital discharge & $122.17 \pm 10.95$ & $121.89 \pm 9.62$ & 0.106 & 0.916 \\
\hline
\end{tabular}

Data are shown as the mean $\pm \mathrm{SD},{ }^{*}$ statistically significant.

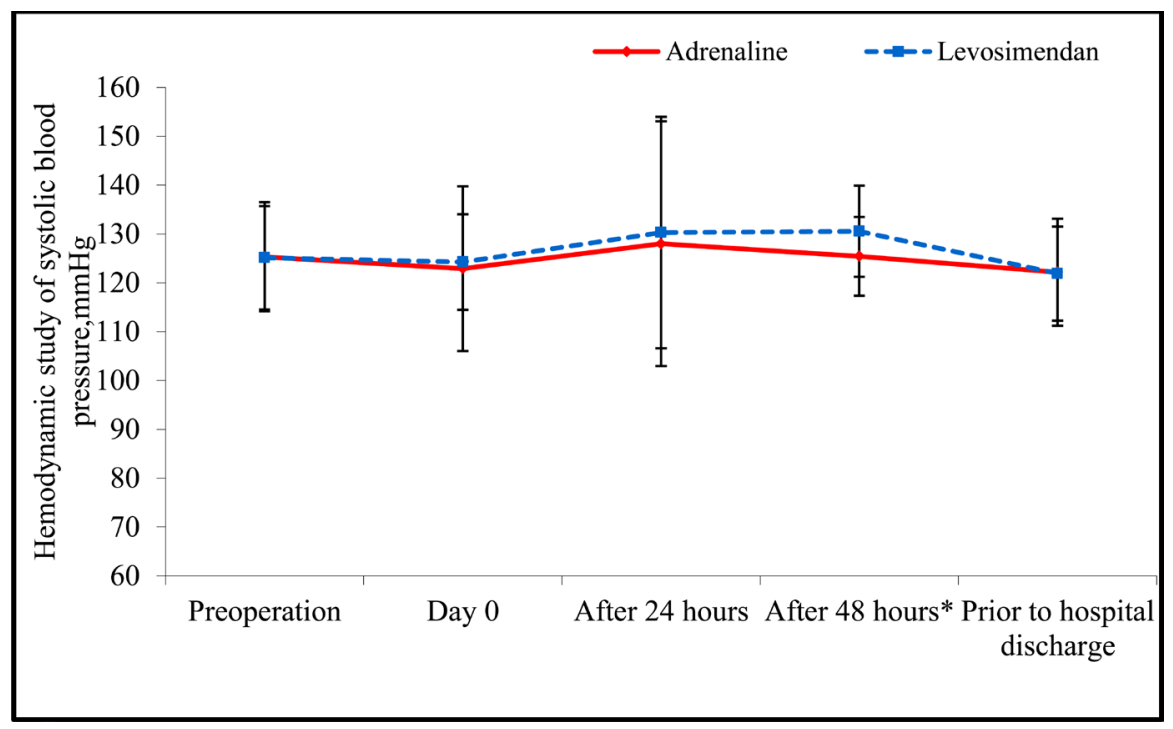

Figure 2. Hemodynamic comparative study of systolic blood pressure, ${ }^{\star}$ statistically significant.

Table 4. Hemodynamic comparative study of diastolic blood pressure (mmHg).

\begin{tabular}{ccccc}
\hline $\begin{array}{c}\text { Hemodynamic study of } \\
\text { diastolic blood pressure }\end{array}$ & Adrenaline & Levosimendan & $\mathrm{t}$ & P-value \\
\hline Preoperation & $76.05 \pm 10.46$ & $75.67 \pm 8.37$ & 0.156 & 0.876 \\
Day 0 & $75.82 \pm 10.69$ & $74.59 \pm 9.45$ & 0.477 & 0.634 \\
After 24 hours & $74.56 \pm 9.26$ & $74.17 \pm 8.62$ & 0.171 & 0.864 \\
After 48 hours & $74.25 \pm 8.68$ & $73.98 \pm 7.28$ & 0.132 & 0.895 \\
Prior to hospital discharge & $73.66 \pm 8.58$ & $72.57 \pm 7.94$ & 0.518 & 0.607 \\
\hline
\end{tabular}

Data are shown as the mean $\pm \mathrm{SD}$.

altering levels of intracellular calcium, which is readily available to sarcoplasmic reticulum [8].

Catecholamines are most widely used in the clinic. Catecholamines exert their cardiac inotropic effect and peripheral vasoconstrictor effect through alpha- and beta-adrenergic receptors $(\mathrm{a} 1, \mathcal{B} 1$, and $\mathcal{B})$. The cardiac effect is mediated through $\mathcal{B} 1$ adrenergic receptors, which when bound to adrenaline, increase the 
levels of intracellular calcium through L-type calcium channels, thereby increasing both the rate and force of contraction [8].

Adrenaline is a $\mathcal{B} 1$ agonist in low doses and an a1 agonist in high doses; thus,

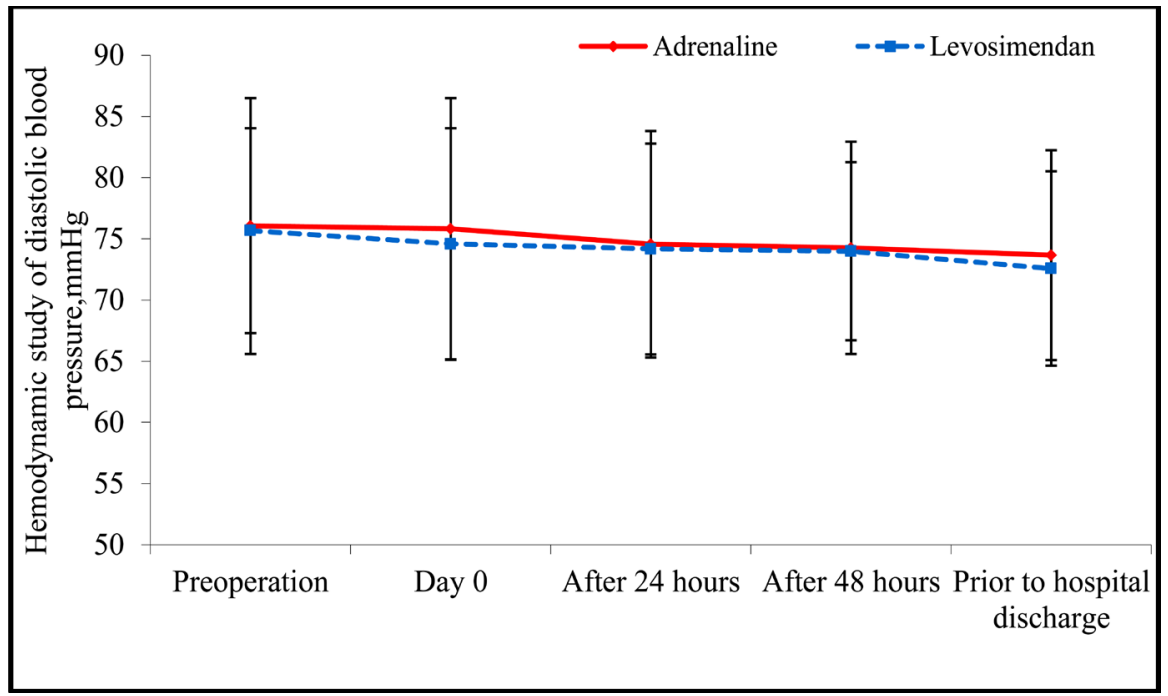

Figure 3. Hemodynamic comparative study of diastolic blood pressure.

Table 5. Comparative study between two groups of patients with an $\mathrm{EF} \leq 30 \%$.

\begin{tabular}{ccccc}
\hline $\mathrm{EF} \leq 30 \%$ & Adrenaline $\mathrm{n}=11$ & Levosimendan $\mathrm{n}=14$ & $\mathrm{X}^{2} / \mathrm{t}$ & P-value \\
\hline Preoperative IABP & 5 & 5 & 0.149 & 0.700 \\
Postoperative IABP & 1 & 1 & 0.026 & 0.872 \\
Adjuvant adrenaline use & Not applicable & 14 & - & - \\
Adrenaline dose (ng/kg/min) & $264.58 \pm 30.29$ & $173.59 \pm 21.37$ & 13.435 & $<0.001^{* *}$ \\
Adjuvant noradrenaline use & 4 & Not applicable & - & - \\
Noradrenaline dose (ng/kg/min) & $177.62 \pm 21.57$ & $123.48 \pm 17.65$ & 10.714 & $<0.001^{* *}$ \\
Death & 2 & 1 & 0.158 & 0.691
\end{tabular}

Data are shown as the mean $\pm \mathrm{SD}$, IABP $=$ intra-aortic balloon pump, ${ }^{* *}$ statistically highly significant.

Table 6. Comparative study between two groups of patients an EF $>30 \%$.

\begin{tabular}{ccccc}
\hline EF $>30 \%$ & Adrenaline $\mathrm{n}=24$ & Levosimendan $\mathrm{n}=14$ & $\mathrm{X}^{2} / \mathrm{t}$ & P-value \\
\hline Preoperative IABP & 1 & 0 & 0.813 & 0.367 \\
Postoperative IABP & 3 & 0 & 2.520 & 0.112 \\
Adjuvant adrenaline use & Not applicable & 6 & - & - \\
Adrenaline dose (ng/kg/min) & $159.37 \pm 24.95$ & $112.48 \pm 21.37$ & 7.892 & $<0.001^{* *}$ \\
Adjuvant noradrenaline use & 2 & Not applicable & - & - \\
Noradrenaline dose (ng/kg/min) & $164.38 \pm 23.48$ & $127.38 \pm 18.6$ & $6.801<0.001^{* *}$ \\
Death & 2 & 0 & 0.315 & 0.575 \\
\hline
\end{tabular}

Data are shown as the mean $\pm \mathrm{SD}, \mathrm{IABP}=$ intra-aortic balloon pump, ${ }^{* *}$ statistically highly significant. 


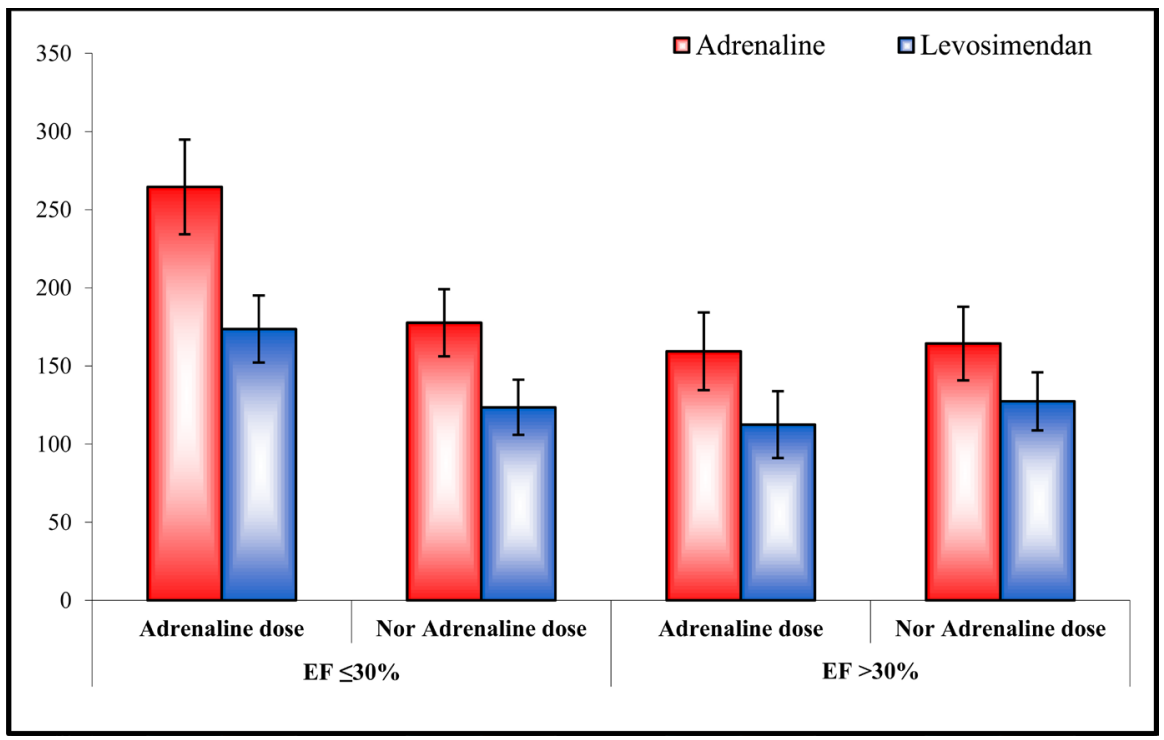

Figure 4. Adrenaline and noradrenaline doses in the two groups according to their preoperative EF.

high doses are not preferred in patients with a metabolic profile of hyperlactatemia and hyperglycemia. In addition, high doses of adrenaline have undesirable effects of tachycardia in ischemic patients [9].

In contrast, levosimendan has a relatively more favorable metabolic profile. Levosimendan increases myocardial contractility without increasing oxygen demand and "unfavorable" tachycardia thorough sensitization of troponin $\mathrm{C}$ to calcium, thus enhancing the binding of troponin $\mathrm{C}$ to calcium and increasing myocardial contractility [10].

Rungatscher and colleagues showed in their animal model the superiority of levosimendan over adrenaline in improving myocardial contractility during the rewarming stage after deep hypothermic cardiopulmonary bypass. The researchers successfully shed light on $B$ adrenergic receptor function during the pathophysiologic conditions of hypothermia. The function of $\mathcal{B}$ adrenergic receptors tends to markedly diminish during hypothermia, leading to the use of higher doses of catecholamines with the subsequent increase in oxygen demand, arrhythmia and regional hypoperfusion leading to organ damage [11].

Lilleberg et al. conducted the first human randomized clinical trial (RCT) to evaluate the efficacy and safety of levosimendan. The trial consisted of low-risk patients with a normal ejection fraction who were undergoing isolated CABG. The patients showed marked improvement in myocardial functions without a significant association with tachycardia and myocardial oxygen demand [12]. Nijhawan and colleagues confirmed these data [13].

In our special category of patients with depressed LV functions, Rajek and colleagues were among the first authors who reported "dramatic" improvement of cardiac output after CBP, minimizing inotropic support requirements and decreasing the overall duration of ICU stay [14]. Many other authors have confirmed their findings [15]. 
Raja and colleagues concluded in their meta-analysis that levosimendan indeed increased myocardial performance with a reduction in afterload. They also recommended the use of levosimendan in the preoperative period to decrease the need for postoperative catecholamine treatment, mechanical support and/or an ICU stay [15].

In 2017, Sanfilippo and colleagues published their meta-analysis of six RCTs, including patients with an EF below 35\% who were undergoing various cardiac operations. The researchers demonstrated a significant reduction in mortality in patients with severe LV dysfunction without affecting overall "all-cause" mortality [16].

Many authors demonstrated a "better timing" of levosimendan administration to minimize myocardial damage and the need for inotropic support, vasopressors and mechanical support. Most researchers agree that preoperative administration of levosimendan 12-24 hours before CPB is beneficial [17] [18].

To assess the "potential benefit" of preoperative levosimendan administration, a number of RCTs were initiated in a multicenter approach: the LEVO-CTS trial, the CHEETAH trial and the Levosimendan in Coronary Artery Revascularization (LICORN) trial [19].

The Levosimendan in Patients with Left Ventricular Dysfunction Undergoing Cardiac Surgery (LEVO-CTS) trial results were published in 2016. The study concluded that prophylactic use of levosimendan did not improve the outcomes of mortality, perioperative myocardial infarction or mechanical support when compared to placebo [20].

The Levosimendan for Hemodynamic Support after Cardiac Surgery (CHEETAH) trial was stopped, as the efforts were deemed "futile". After enrolling 506 patients, no statistically significant difference was found between the low-dose levosimendan group and placebo group when combined with standard ICU care protocols. There were also no differences in 30-day-hospital mortality, mechanical ventilation, low cardiac output syndrome (LCOS) and dysrhythmias with levosimendan treatment compared to placebo drug treatment [21].

The most recent clinical trial investigating the role of levosimendan, titled "Effect of Levosimendan on Low Cardiac Output Syndrome in Patients With Low Ejection Fraction Undergoing Coronary Artery Bypass Grafting With Cardiopulmonary Bypass: The LICORN Randomized Clinical Trial”, published their results late in 2017. Levosimendan, when compared to placebo, failed to induce a significant difference in terms of mortality, the duration of inotropic support use and the duration of mechanical support use in patients with depressed left ventricular function undergoing CABG. Thus, the use of levosimendan as a prophylactic drug was not recommended in this category of patients [22].

The current study concluded that levosimendan use may be associated with a lower incidence of postoperative arrhythmia, less need for mechanical support, less mechanical ventilation hours, and shorter durations of ICU stay than adrenaline use. The hemodynamic response, dose and period of inotropic and vaso- 
constrictor use were variable in the two groups. The primary outcome for this study showed no statistically significant difference between the two pharmacological protocols.

\section{Limitations of the Study}

The present study is limited by its retrospective design and small population. In addition, the exclusion of associated ischemic mitral regurgitation pathology and/or intervention that significantly affects the outcome in patients with a low EF is another limitation. Additionally, the study lacks follow-up data for the assessment of mid- and late-term results and outcomes.

\section{Conclusion}

The use of levosimendan in low ejection fraction patients undergoing CABG did not alter the overall mortality. However, levosimendan treatment decreases the use of adjuvant inotropic support that may be needed in such cases and may be hazardous if used in high doses. Additionally, levosimendan treatment may further decrease the ventilation hours and the duration of ICU stay. This study paves the way for further research to establish an optimized protocol for the management of such a challenging condition.

\section{Conflicts of Interest}

The authors confirm that they have no competing interests or any financial or commercial affiliations to disclose.

\section{References}

[1] Diodato, M. and Chedrawy, E.G. (2014) Coronary Artery Bypass Graft Surgery: The Past, Present, and Future of Myocardial Revascularisation. Surgery Research and Practice, 2014, 1-6. https://doi.org/10.1155/2014/726158

[2] Cesar, L.A.M. and Viana, C.B. (2014) Strategy for Therapeutic Decision Medical vs. Angioplasty vs. Surgery. Revista da Sociedade de Cardiologia do Estado de São Paulo, 24, 17-22.

[3] Kamal, Y.A., Al-Elwany, S.E., Ghoneim, A.M. and El-Minshawy, A.M. (2017) Predictors of Adverse Effects after Coronary Artery Bypass Grafting in Patients with Reduced Left Ventricular Ejection Fraction. Journal of the Egyptian Society of Cardio-Thoracic Surgery, 25, 20-27. https://doi.org/10.1016/j.jescts.2017.02.002

[4] Deppe, A.-C., Weber, C., Liakopoulos, O.J., et al. (2017) Preoperative Intra-Aortic Balloon Pump Use in High-Risk Patients Prior to Coronary Artery Bypass Graft Surgery Decreases the Risk for Morbidity and Mortality-A Meta-Analysis of 9,212 Patients. Journal of Cardiac Surgery, 32, 177-185. https://doi.org/10.1111/jocs.13114

[5] Ibanez, B., James, S., Agewall, S., Antunes, M.J., Bucciarelli-Ducci, C., Bueno, H., Caforio, A.L.P., Crea, F., Goudevenos, J.A., Halvorsen, S., Hindricks, G., Kastrati, A., Lenzen, M.J., Prescott, E., Roffi, M., Valgimigli, M., Varenhorst, C., Vranckx, P. and Widimský, P. (2017) ESC Guidelines for the Management of Acute Myocardial Infarction in Patients Presenting with ST-Segment Elevation: The Task Force for the Management of Acute Myocardial Infarction in Patients Presenting with STSegment Elevation of the European Society of Cardiology (ESC). European Heart 
Journal, ehx393.

[6] Hamad, M.A.S., Straten, A.H.V., Schönberger, J.P., et al. (2010) Preoperative Ejection Fraction as a Predictor of Survival after Coronary Artery Bypass Grafting: Comparison with a Matched General Population. Journal of Cardiothoracic Surgery, 5.

[7] Pieri, M., Belletti, A., Monaco, F., et al. (2016) Outcome of Cardiac Surgery in Patients with Low Preoperative Ejection Fraction. BMC Anesthesiology, 16. https://doi.org/10.1186/s12871-016-0271-5

[8] Berry, W. and McKenzie, C. Use of Inotropes in Critical Care. Pharmaceutical Journal.

https://www.pharmaceutical-journal.com/learning/learning-article/use-of-inotropes -in-critical-care/11049283.article

[9] Dickstein, K. (2008) ESC Guidelines for the Diagnosis and Treatment of Acute and Chronic Heart Failure 2008: Application of Natriuretic Peptides: Reply. European Heart Journal, 30, 383. https://doi.org/10.1093/eurheartj/ehn561

[10] Ukkonen, H. (2000) Myocardial Efficiency during Levosimendan Infusion in Congestive Heart Failure. Clinical Pharmacology \& Therapeutics, 68, 522-531. https://doi.org/10.1067/mcp.2000.110972

[11] Rungatscher, A., Linardi, D., Tessari, M., et al. (2012) Levosimendan Is Superior to Epinephrine in Improving Myocardial Function after Cardiopulmonary Bypass with Deep Hypothermic Circulatory Arrest in Rats. The Journal of Thoracic and Cardiovascular Surgery, 143, 209-214. https://doi.org/10.1016/j.jtcvs.2011.09.020

[12] Lilleberg, J., Nieminen, M.S., Akkila, J., Heikkila, L., Kuitunen, A. and Lehtonen, L. (1998) Effects of a New Calcium Sensitizer Levosimendan on Haemodynamics, Coronary Blood Flow and Myocardial Substrate Utilization Early after Coronary Artery Bypass Grafting. European Heart Journal, 19, 660-668. https://doi.org/10.1053/euhj.1997.0806

[13] Nijhawan, N., Nicolosi, A.C., Montgomery, M.W., Aggarwal, A., Pagel, P.S. and Warltier, D.C. (1999) Levosimendan Enhances Cardiac Performance after Cardiopulmonary Bypass: A Prospective, Randomized Placebo-Controlled Trial. Journal of Cardiovascular Pharmacology, 34, 219-228. https://doi.org/10.1097/00005344-199908000-00007

[14] Rajek, A.M., Koinig, H., Jelen, M., Schiferer, A. and Hutschala, D. (2003) Levosimendan, a New Ca-Sensitizer, in Patients with Poor Left Ventricular Function Undergoing Cardiac Surgery. Anesthesiology, 99, A133.

[15] Raja, S.G. and Rayen, B.S. (2006) Levosimendan in Cardiac Surgery: Current Best Available Evidence. The Annals of Thoracic Surgery, 81, 1536-1546.

https://doi.org/10.1016/j.athoracsur.2005.08.073

[16] Sanfilippo, F., Knight, J.B., Scolletta, S., et al. (2017) Levosimendan for Patients with Severely Reduced Left Ventricular Systolic Function and/or Low Cardiac Output Syndrome Undergoing Cardiac Surgery: A Systematic Review and Meta-Analysis. Critical Care, 21, 252. https://doi.org/10.1186/s13054-017-1849-0

[17] Eris, C., Yavuz, S., Toktas, F., Turk, T., Gucu, A., Erdolu, B. and Goncu, M.T. (2014) Preoperative Usages of Levosimendan in Patients Undergoing Coronary Artery Bypass Grafting. International Journal of Clinical and Experimental Medicine, 7, 219-229.

[18] Tritapepe, L., Santis, V.D., Vitale, D., et al. (2009) Levosimendan Pre-Treatment Improves Outcomes in Patients Undergoing Coronary Artery Bypass Graft Surgery. British Journal of Anaesthesia, 102, 198-204. https://doi.org/10.1093/bja/aen367 
[19] Nenna, A., Greco, S.M., Barbato, R. and Chello, M. (2017) Levosimendan in Cardiac Surgery: Is It All Gold That Glitters? Journal of Cardiovascular Diseases \& Diagnosis, 5, 276. https://doi.org/10.4172/2329-9517.1000276

[20] Mehta, R.H., Leimberger, J.D., Diepen, S.V., et al. (2017) Levosimendan in Patients with Left Ventricular Dysfunction Undergoing Cardiac Surgery. New England Journal of Medicine, 376, 2032-2042. https://doi.org/10.1056/NEJMoa1616218

[21] Landoni, G., Lomivorotov, V.V., Alvaro, G., et al. (2017) Levosimendan for Hemodynamic Support after Cardiac Surgery. New England Journal of Medicine, 376, 2021-2031. https://doi.org/10.1056/NEJMoa1616325

[22] Cholley, B., Caruba, T., Grosjean, S., Amour, J., Ouattara, A., Villacorta, J., Miguet, B., Guinet, P., Lévy, F., Squara, P., Aït Hamou, N., Carillon, A., Boyer, J., Boughenou, M., Rosier, S., Robin, E., Radutoiu, M., Durand, M., Guidon, C., Desebbe, O., Charles-Nelson, A., Menasché, P., Rozec, B., Girard, C., Fellahi, J., Pirracchio, R. and Chatellier, G. (2017) Effect of Levosimendan on Low Cardiac Output Syndrome in Patients with Low Ejection Fraction Undergoing Coronary Artery Bypass Grafting with Cardiopulmonary Bypass the Licorn Randomized Clinical Trial. JAMA, 318, 548-556. https://doi.org/10.1001/jama.2017.9973 